\title{
Da distribuição de frequências de terremotos globais registrados na base USGS entre 2003 e 2017
}

\author{
On the frequency distribution of world earthquakes registered in the USGS database \\ between 2003 and 2017
}

João Henrique Rettore Totaro Geólogo, Professor do Programa de Pós-Graduação em Geografia da PUC Minas, Brasil infoespa@pucminas.br

\begin{abstract}
Resumo
O presente artigo compila os resultados obtidos na primeira fase de projeto que investiga as correlações entre diversos parâmetros descritivos de eventos sísmicos seguindo uma linha teóricometodológica relativamente recente na sismologia, a qual implica o tratamento estatístico de massas de dados para a identificação de padrões espaciais e temporais de distribuição de terremotos. Desenvolvem-se, ao longo do trabalho, argumentos que indicam tanto a existência de faixas cuja sismicidade é mais variável quanto uma mudança na sismicidade global ocorrida no período de amostragem.
\end{abstract}

Palavras-chave: sismicidade global, bases de dados eletrônicas, padrões espaciais e temporais

\begin{abstract}
This article presents a compilation of the numerical results obtained in the first phase of a project that investigates the correlations between several descriptive parameters os seismic events according to a relatively recent theoretical and methodological approach in seismology, which involves the statistical treatment of large databases for the identification of spatial and temporal patterns of distribution of earthquakes. Throughout the paper, arguments indicate both the existence of regions in which seismicity is more variable and a change in the global seismicity pattern that took place in the sampling interval.
\end{abstract}

Keywords: global seismicity, electronic databases, spatial and temporal patterns

\section{INTRODUÇÃO}

A sismicidade mundial, ainda que majoritariamente associada a estruturas litosféricas ativas que atingem grande profundidade, exibe diversas características de um sistema complexo (TURCOTTE; MALAMUD, 2002): comportamento emergente, baixa previsibilidade e dinâmica não-linear são apenas algumas delas. Seu concurso leva à identificação de padrões de dispersão de energia mecânica cuja variação complexa está diretamente associada à dinâmica dos fragmentos litosféricos, determinada, por sua vez, pelo regime convectivo da astenosfera.

Este trabalho está inserido em projeto mais amplo, conduzido na PUC Minas, e que pretende oferecer uma aproximação à verificação da lei de Gutenberg-Richter (1949) para a relação entre a 
magnitude e o número total de terremotos, a partir de dados colhidos na plataforma do USGS ${ }^{1}$ referente ao período 2003-2017. Nesta etapa, orientamo-nos pela determinação da distribuição de sismos feita por Ballore (1906) e desenvolvida por Heck (1935) e Rothé (1969), introduzindo aqui a proposta de estudo dessa distribuição a partir de faixas de latitude. Essa setorização implica, a nosso ver, uma artificialidade menor que a setorização tradicional em massas continentais ou segundo argumentos geopolíticos, pois não apenas a inércia rotacional em latitudes distintas leva a diferentes regimes na astenosfera (SIDORENKOV, 2009), mas também os contatos entre uma mesma placa e cada uma de suas circundantes são associados a efeitos diversos. Exemplo claro desse ponto são os extensos cinturões sísmicos do Pacífico, que apresentam áreas de atividade heterogênea. Há que fazer ainda uma concessão adicional à proposta, haja vista a existência de zonas intensamente sísmicas cuja orientação principal segue os paralelos geográficos, como as que se estendem a E do Mediterrâneo Oriental. Não obstante o exposto, há claras evidências de que o método é eficaz na identificação de padrões de comportamento sísmico em faixas específicas, como será demonstrado.

A ocorrência de sismos de diversas magnitudes está preferencialmente associada a cinturões bem delimitados (Figuras 1 e 2), de ordinário acompanhados de vulcanismo ativo nas áreas de convergência ou de divergência de frações da litosfera. Por certo, as grandes faixas tectônicas que se sobrepõem a regiões continentais (orientadas segundo eixos gerais E-W, no bloco eurasiático, e N-S / NW - SE, para as Américas) se compõem de frações de atividade sísmica heterogênea. A moderna instrumentação sismológica permite a detecção de eventos sísmicos, naturais ou antropogênicos, de magnitudes negativas: há registros de detecção de sismos naturais de até $-4 \mathrm{M}_{\mathrm{L}}$ com o auxílio de sismógrafos Wood-Anderson ${ }^{2}$, o que estende muitíssimo o alcance da teoria em termos da compreensão dos mecanismos regidos pela cessão de materiais rochosos em domínio rúptil e das relações entre eventos de magnitudes diversas.

Ogata e Katsura (1993) conduzem uma análise espacial e temporal da distribuição de frequência de terremotos a partir do exame de catálogos de sismos ocorridos no Japão no intervalo de 1926 a 1990; esses autores estudam a correlação entre magnitudes e frequência, e sugerem uma elevação na frequência de sismos de baixa magnitude a acompanhar o incremento da densidade das redes locais e integradas de monitoração sísmica. As relações entre magnitude e frequência são apropriadamente abordadas por Bullen e Bolt (1985) a partir da equação de Gutenberg e Richter ${ }^{3}$, que se aplica a eventos globais de qualquer magnitude.

1 earthquake.usgs.gov

2 The National Academies. Induced seismicity potential in energy technologies. Washington, DC: The National Academies Press, 2013.

3 Expressamente, $\log \mathbf{N}=\mathbf{a}-\mathbf{b M s}$, em que $\mathbf{N}$ é o número médio de eventos por ano, $\mathbf{a}$ é a 'produtividade' ou grau de atividade sísmica e b está associada ao ambiente tectônico $(1.8 \leq \mathrm{b} \leq 1.0$ para cristas mesooceânicas, $1.0 \leq \mathrm{b} \leq 0.7$ para seções interplacas e $0.7 \leq \mathrm{b} \leq 0.4$ para faixas intraplacas). A relação foi empiricamente definida por B. Gutenberg e C. F. Richter em 1935(1949). 


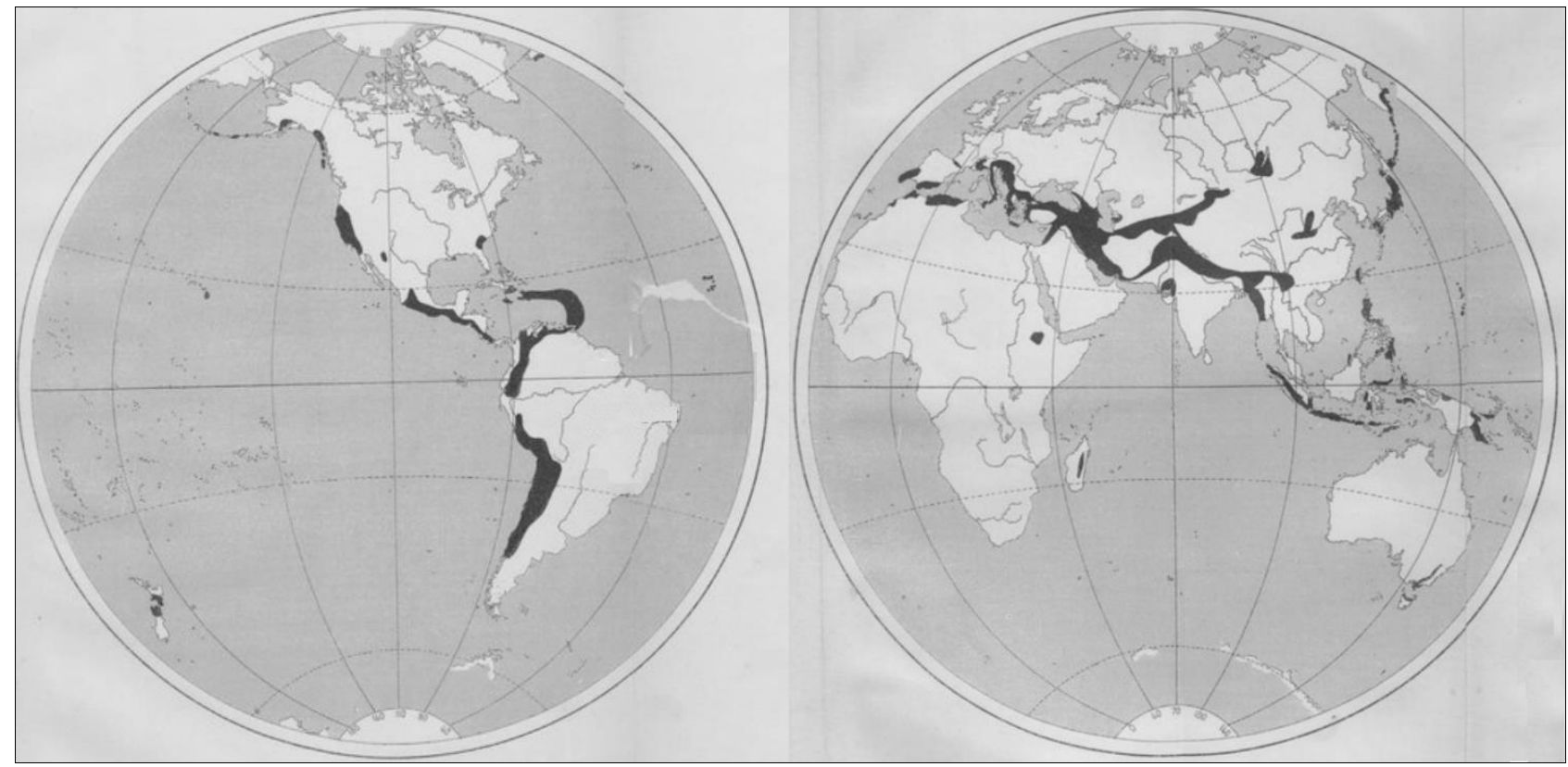

Figura 1 - Identificação das principais regiões sísmicas do globo (faixas mais escuras) por M. de Ballore (1906).

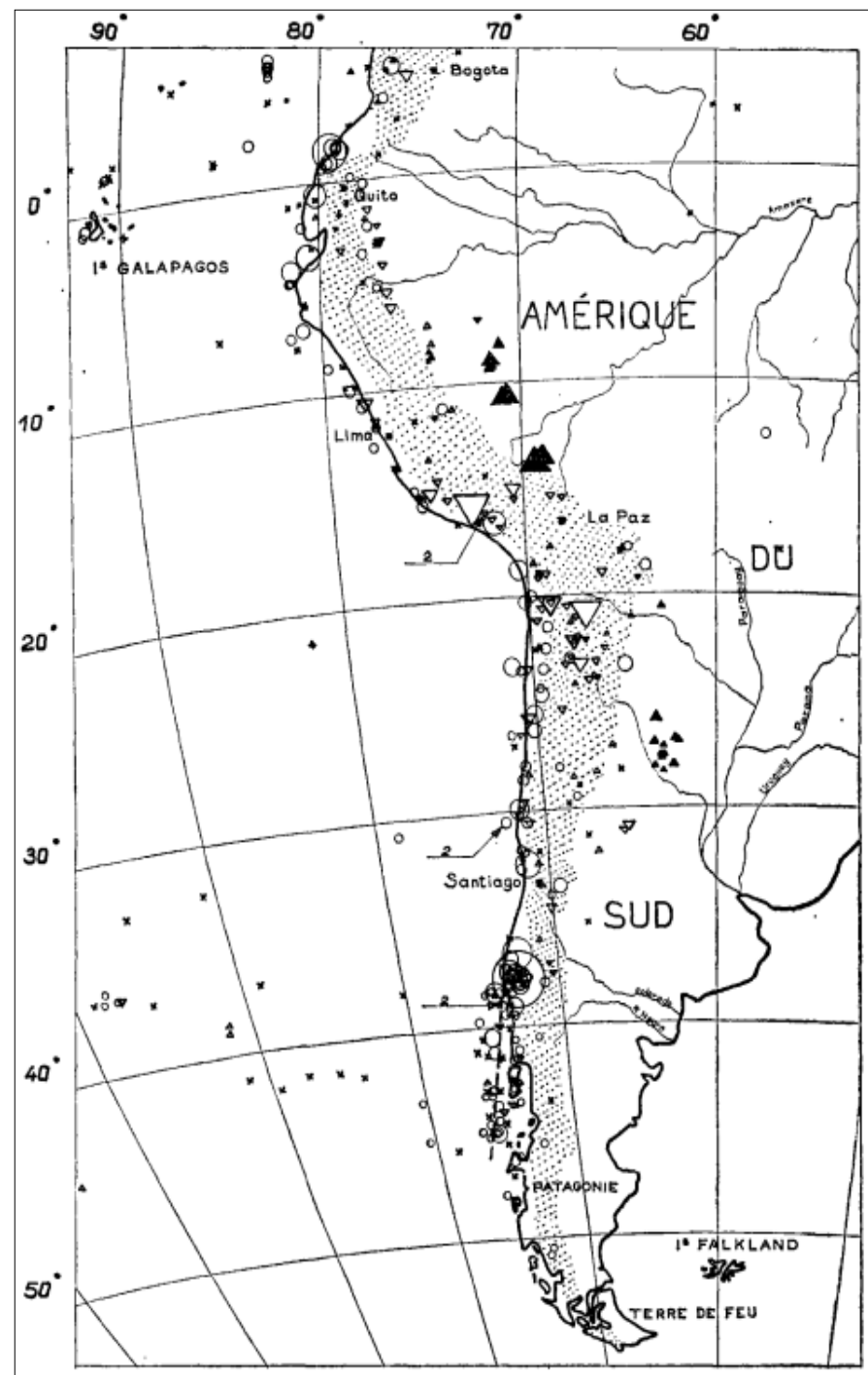

Figura 2 - Localização de epicentros de terremotos no período 1953-1965 para a América do Sul (Rothé 1969). A magnitude de cada evento é indicada pela dimensão de círculo ou triângulo a ele associado; as faixas de profundidade focal são indicadas por círculos vazios (rasos), triângulos vazios (intermediários) ou triângulos cheios (profundos). 
Obviamente, a localização de epicentros de terremotos é apenas um dos muitos parâmetros essenciais à correta compreensão do mecanismo gerador desses fenômenos e, em última análise, à caracterização de todos os seus desdobramentos. Descritores recorrentes nas bases de dados sismológicas são também a profundidade do foco, o tipo de magnitude e elementos técnicos (fases, sismogramas de cada estação etc.), e a ampliação das bases de informações sismológicas é imprescindível para a compreensão das várias correlações possíveis entre esses eventos (McCaffrey 2008).

Além do USGS, outras fontes de dados oferecem informações sobre terremotos; algumas delas são:

- EMSC (European-Mediterranean Seismological Centre).

https://www.emsc-csem.org/\#2

Catálogo de terremotos globais e da faixa mediterrânea desde 2004; filtros por magnitude e recentidade para os mapas interativos. Informações técnicas (diversas soluções para tensores de momento); link para o EPOS (European Plate Observing System), que traz informações sobre terremotos registrados desde 1998 e coordena estudos sobre geotectônica, e outras redes, projetos e portais de informações afins.

- IRIS (Incorporated Research Institutions for Seismology)

http://www.iris.edu/hq/

Sismogramas, mapas bi- ou tridimensionais interativos; filtros por localização, magnitude, intervalo de tempo, profundidade focal. O catálogo recua até o início da década de 1970 e conta com mais de $5.4 \mathrm{M}$ registros.

- ISC (International Seismological Centre)

http://www.isc.ac.uk/iscbulletin/search/bulletin/

Catálogo organizado a partir de 2015, incluindo sismogramas para cada evento. A busca por informações pode ser feita segundo faixas de Flinn-Engdahl, setores circulares ou regiões retangulares, além dos elementos descritivos dos sismos.

- NOAA (National Oceanic and Atmospheric Administration) - National Centers for Environmental Information.

https://www.ngdc.noaa.gov/hazard/earthqk.shtml

Catálogo de terremotos significativos ocorridos desde 2150a.C. até o presente, numa lista dinâmica que conta, até o momento, com 5997 entradas; a busca por informações pode ser definida segundo critérios geográficos, sismológicos (magnitude, intensidade, profundidade do hipocentro), efeitos secundários (tsunamis, seiches, erupções vulcânicas) e organizada a partir de diversos elementos descritivos.

Catálogo de erupções vulcânicas significativas desde 1750a.C., que podem ser pesquisadas segundo a classe estrutural do vulcão (caldeira, escudo, maar etc.), sua localização, VEI e efeitos secundários (terremotos ou tsunamis associados).

Catálogo de tsunamis ocorridos desde 2000a.C. nos oceanos Atlântico, Índico e Pacífico e nos mares Mediterrâneo e do Caribe, organizado segundo áreas fonte e afetada, magnitude do terremoto deflagrador e elementos descritivos (inundação horizontal, altura máxima em terra etc.). 
Há também numerosos catálogos locais, cujo incremento em termos de volume de informação tem crescido rapidamente ${ }^{4}$; apesar de não ser nosso propósito fazer uma lista exaustiva dessas fontes, destacamos:

- Centro Sismológico Nacional - Universidade de Chile

http://www.sismologia.cl/

Dados sobre sismos locais ocorridos desde 2006; filtros por intervalo de tempo, localização, magnitude e profundidade do hipocentro; inclui perfis sísmicos que demonstram claramente a distribuição dos focos em padrão que acompanha a subducção da placa de Nazca.

- ЗЕМЛЕТРЯСЕНИЯ КАМЧАТКИ (Earthquake in Kamtchatka)

http://quakekam.emsd.ru/

Catálogo de sismos ocorridos na Península de Kamtchatka desde 1996; filtros por intervalo de tempo, localização, magnitude e profundidade do foco.

- Erdbebenkatalog - Universität zu Köln

http://www.seismo.uni-koeln.de/catalog/2012.htm

Dados registrados pela estação de Bensberg, Alemanha, desde 1975.

- Icelandic Met Office

http://en.vedur.is/earthquakes-and-volcanism/earthquakes/

Mapa interativo de terremotos ocorridos na Islândia nas últimas 48h; medidas de strain local; catálogo de terremotos locais (magnitude $\geq 4.0$ ) desde 1706 .

- Instituto Português do Mar e da Atmosfera

http://www.ipma.pt/en/geofisica/sismologia/

Informações sobre terremotos globais e locais ocorridos desde 1998; detalhamento da rede sísmica portuguesa, tanto continental quanto insular.

- Istituto Nazionale di Geofisica e Vulcanologia

http://www.ct.ingv.it/ufs/analisti/catalogolist.php

Dados sobre terremotos registrados pela rede sísmica da Itália meridional, especialmente para a Sicília e a Calábria, desde 1999.

- Iranian Seismological Center (Inst. Geophysics / Univ. Tehran)

http://irsc.ut.ac.ir/currentearthq.php

Informações técnicas detalhadas (mecanismos focais, registros de fase, profundidade focal) dos últimos 60 terremotos ocorridos no Irã; catálogo de eventos locais e regionais pré-históricos até 2000.

- National Earthquake Information Database https://www.gns.cri.nz/Home/Products/Databases/National-Earthquake-Information-Database Catálogo de eventos sísmicos, mapas e informações gerais sobre terremotos ocorridos na Nova Zelândia desde 2010.

4 Comparem-se, por exemplo, as perspectivas oferecidas por V. B. Smirnof - Earthquake catalogs: evaluation of data completeness - Volc. Seis., 1988, vol. 19, pp. 497-510 e por Enzo Boschi - A 'new generation' earthquake catalogue - Annali di Geofisica, vol. 43, n. 4, Aug. 2000. 
A proposta aqui desenvolvida não contempla conjuntos de eventos relacionados em termos processuais, como é o caso de terremotos precursores (foreshocks) e de réplicas (aftershocks), além disso, sismos artificiais são computados em virtude de seu potencial papel na deflagração de processos regionais. Dessa forma, evitam-se os problemas associados a agrupamentos (clusters) em catálogos sismológicos, como discutido por Kagan (2014) em termos da cobertura espacial das redes sismográficas - especialmente em relação a microssismos - e à adequação de modelos estocásticos fundamentados na teoria de processos de ramificação (KAGAN, 2006). Esse último caso é fortemente afetado por fatores como o alcance e a sensibilidade das redes sismográficas, a geometria diferencial dessas redes e mesmo a precisão das mais antigas para a determinação de coordenadas focais e magnitudes (KAGAN, 2014, p.13). Por fim, não é considerado aqui o papel de mecanismos geofísicos cuja demonstração ainda não é bem estabelecida, como a proposta de correlação entre sismicidade global e oscilações esferoidais fundamentais ${ }_{0} \mathrm{~S}_{2}$ (GUGLIELMI; ZOTOV 2012).

Ordinariamente, as bases USGS comportam informações detalhadas sobre cada evento registrado na rede sísmica mundial, e os elementos técnicos específicos oferecidos pelo portal são muitíssimo úteis para a investigação de aspectos teóricos diversos da sismologia. Entre as características técnicas descritas para cada evento há informações de contexto sismotectônico, tensores de momento (incluindo especificações sobre planos nodais e eixos de deslocamento) e distribuição do rejeito em planta e em perfil segundo o plano de ruptura. Os dados oferecidos no portal permitem, portanto, conduzir investigações em variadas linhas complementares à que apresentamos neste trabalho.

\section{METODOLOGIA}

As bases de dados anuais foram colhidas, mês a mês, no site do USGS em formato .CSV (Figura 3, referente ao mês de março/2008) e integradas em planilha eletrônica LibreOffice de maneira que cada ano corresponda a uma pasta. Isso permite o isolamento de variáveis quaisquer e a contagem automática da ocorrência de eventos, além do cruzamento de resultados entre planilhas.

Para este trabalho, os dados anuais foram separados por faixa de latitude, a partir de $90^{\circ} \mathrm{N}$ (assinalado como +90) e a intervalos de $10^{\circ}$, até $90^{\circ} \mathrm{S}$ (limite assinalado como -90), a partir de planilha auxiliar como a apresentada na Figura 4 para o mesmo ano de 2008. Em seguida, procedeuse à contagem simples dos eventos por faixa de latitude ao longo do intervalo de amostragem. A base de dados original permite, portanto, uma série de correlações entre as variáveis, e a significância do conjunto em termos do tratamento estatístico estende grande confiabilidade aos resultados. 


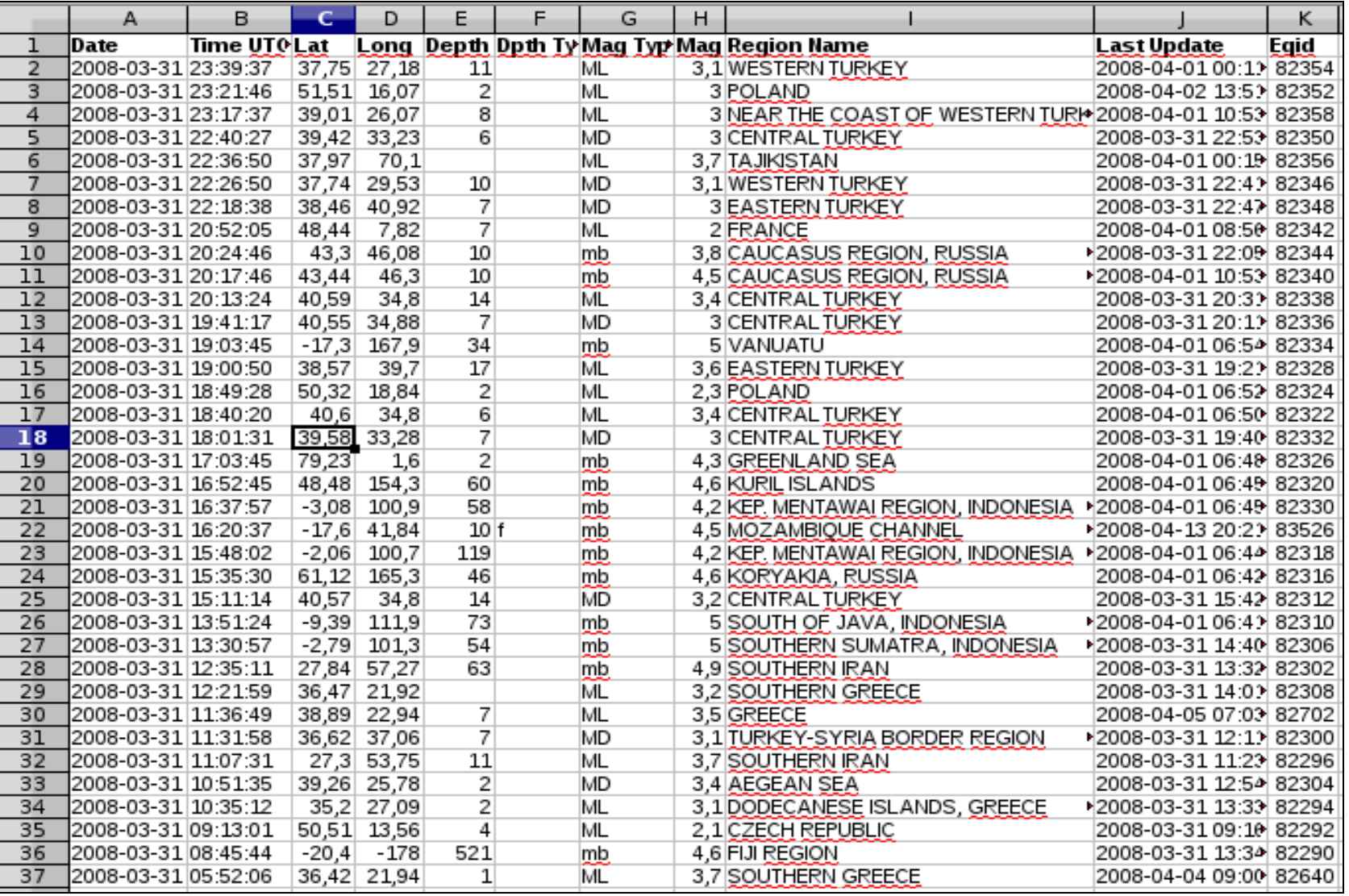

Figura 3 - Tabela de dados brutos gerados pela importação de dados em formato CSV e referente ao ano de 2008 na base USGS. É apenas necessário identificar corretamente, no processo, os separadores de campo que delimitam as informações no arquivo original.

A magnitude dos eventos que compõem a base final de dados é elemento descritivo que, por exigir outras considerações teórico-metodológicas, já está em fase de processamento e será apreciado em outro trabalho, ao longo de considerações mais detalhadas acerca da eventual concentração de sismos em regiões específicas, nos máximos locais indicados no conjunto de dados e sua possível associação com outros fenômenos relacionados aos mecanismos da dinâmica interna.

\section{RESULTADOS E SUA DISCUSSÃO}

Uma vez que os dois conjuntos podem ser resolvidos por funções quadráticas e a $<1$ em (2), a parábola descrita por essa equação é necessariamente mais aberta que a associada a (1), o que também corresponde às diferenças escalares no eixo das ordenadas das Figuras. 4 e 5.

Os máximos locais observados em 2008 e em 2010 no gráfico 1 são devidos aos valores excêntricos de $n$ nos meses de abril desses mesmos anos, indicados no gráfico 2, os quais atingiram 13150 e 16924 registros, respectivamente. É interessante notar que ambos picos foram colhidos na faixa $40^{\circ} \mathrm{N}-30^{\circ} \mathrm{N}$, que compreende não apenas grande parte do S dos EUA (incluindo a California), mas também outras zonas sismicamente ativas, como o Mediterrâneo meridional, Irã, grande parte dos Himalaias e o Japão, além da Cordilheira Mesoatlântica - ou seja, áreas representativas de 
ambientes tectônicos distintos. Isso sugere que o método de recorte das áreas para monitoração sísmica deve ser harmônico com a natureza do fenômeno envolvido para que se tenha coerência argumentativa; por esse motivo, desenvolver-se-á, proximamente, a hipótese de que a setorização dever obedecer a parâmetros de ambientação tectônica.

Outro aspecto digno de nota respeita a magnitude desses eventos mais numerosos ocorridos na faixa em questão: em abril/2008, registraram-se 4029 sismos de magnitude compreendida entre 2 e 1, e 7798 sismos de magnitude compreendida entre 1 e 0 ; em abril/2010, as faixas de interesse em termos de magnitude são 3 a 2 e 2 a 1, com 4676 e 8260 eventos, respectivamente. Uma análise mais detalhada da distribuição dos terremotos registrados na base de dados do USGS em termos de magnitude de momento está compreendida no escopo geral deste projeto.

Os gráficos anuais individualmente apresentados a seguir demonstram a variação na sismicidade global no período de amostragem. As escalas verticais foram normalizadas de sorte a abarcar o máximo registrado para $n\left(40^{\circ} \mathrm{N}<\right.$ lat $\left.<30^{\circ} \mathrm{N}\right)$ em 2010 ; além disso, cada gráfico é acompanhado de uma proposta de solução algébrica de grau $3^{5}$.

5 As equações de cada curva são:

$\begin{array}{lll}2003 & - & \mathrm{f}(\mathrm{x})=25,8402 \mathrm{x} 3-817,1061 \mathrm{x} 2+6927,7859 \mathrm{x}-8574,0229 \\ 2004 & - & \mathrm{f}(\mathrm{x})=35,6100 \mathrm{x} 3-1119,7178 \mathrm{x} 2+9437,3293 \mathrm{x}-11901,4788 \\ 2005 & - & \mathrm{f}(\mathrm{x})=33,6735 \mathrm{x} 3-1065,8693 \mathrm{x} 2+9060,6806 \mathrm{x}-11638,3562 \\ 2006 & - & \mathrm{f}(\mathrm{x})=28,6551 \mathrm{x} 3-914,6422 \mathrm{x} 2+7858,2383 \mathrm{x}-10165,9706 \\ 2007 & - & \mathrm{f}(\mathrm{x})=25,9133 \mathrm{x} 3-831,0619 \mathrm{x} 2+7181,2413 \mathrm{x}-9373,6307 \\ 2008 & - & \mathrm{f}(\mathrm{x})=41,8387 \mathrm{x} 3-1317,0736 \mathrm{x} 2+11140,7516 \mathrm{x}-14586,5752 \\ 2009 & - & \mathrm{f}(\mathrm{x})=32,0863 \mathrm{x} 3-1001,3132 \mathrm{x} 2+8373,0711 \mathrm{x}-10783,9788 \\ 2010 & - & \mathrm{f}(\mathrm{x})=47,3822 \mathrm{x} 3-1475,7549 \mathrm{x} 2+12313,3059 \mathrm{x}-15815,1144 \\ 2011 & - & \mathrm{f}(\mathrm{x})=41,2156 \mathrm{x} 3-1285,9971 \mathrm{x} 2+10759,3541 \mathrm{x}-13961,3562 \\ 2012 & - & \mathrm{f}(\mathrm{x})=39,5441 \mathrm{x} 3-1235,1836 \mathrm{x} 2+10346,4749 \mathrm{x}-13446,8529 \\ 2013- & - & \mathrm{f}(\mathrm{x})=50,6611 \mathrm{x} 3-1540,4095 \mathrm{x} 2+12762,4524 \mathrm{x}-11985,6046 \\ 2014 & - & \mathrm{f}(\mathrm{x})=52,1128 \mathrm{x} 3-1577,9259 \mathrm{x} 2+12369,4391 \mathrm{x}-10280,9886 \\ 2015 & - & \mathrm{f}(\mathrm{x})=51,1619 \mathrm{x} 3-1545,6041 \mathrm{x} 2+12071,0041 \mathrm{x}-9811,7549 \\ 2016 & - & \mathrm{f}(\mathrm{x})=51,2332 \mathrm{x} 3-1550,8530 \mathrm{x} 2+12158,8883 \mathrm{x}-10199,1340 \\ 2017 & - & \mathrm{f}(\mathrm{x})=54,9256 \mathrm{x} 3-1648,8393 \mathrm{x} 2+12762,4774 \mathrm{x}-10228,6046\end{array}$




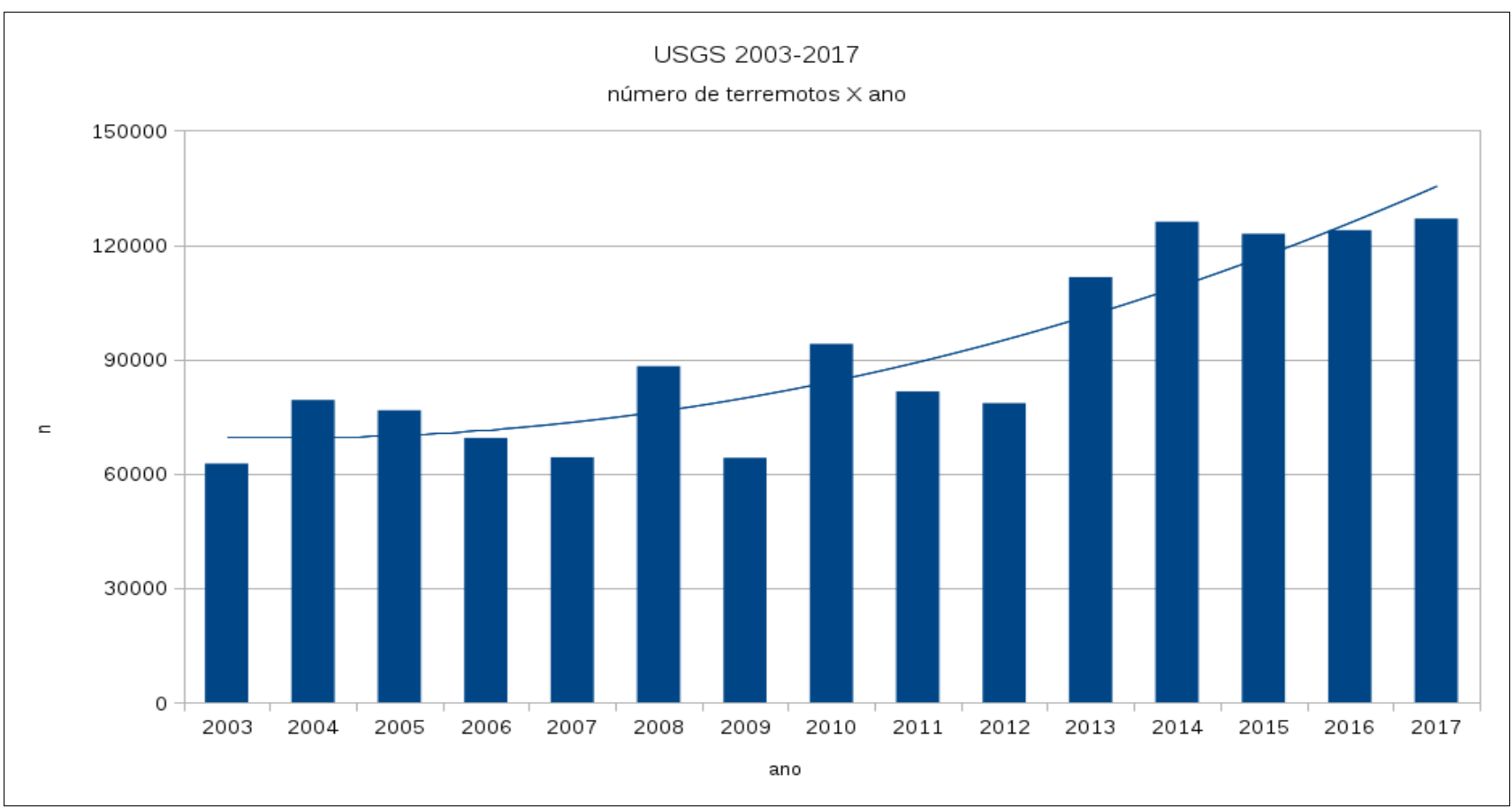

Figura 4 - Distribuição anual de terremotos na base USGS para o período 2003-2017, mostrando clara elevação de n no último terço do intervalo de amostragem; a curva quadrática que representa o comportamento geral dos dados tem equação descrita abaixo.

$f(x)=373,54 x^{2}-1277,08 x+70680,21$

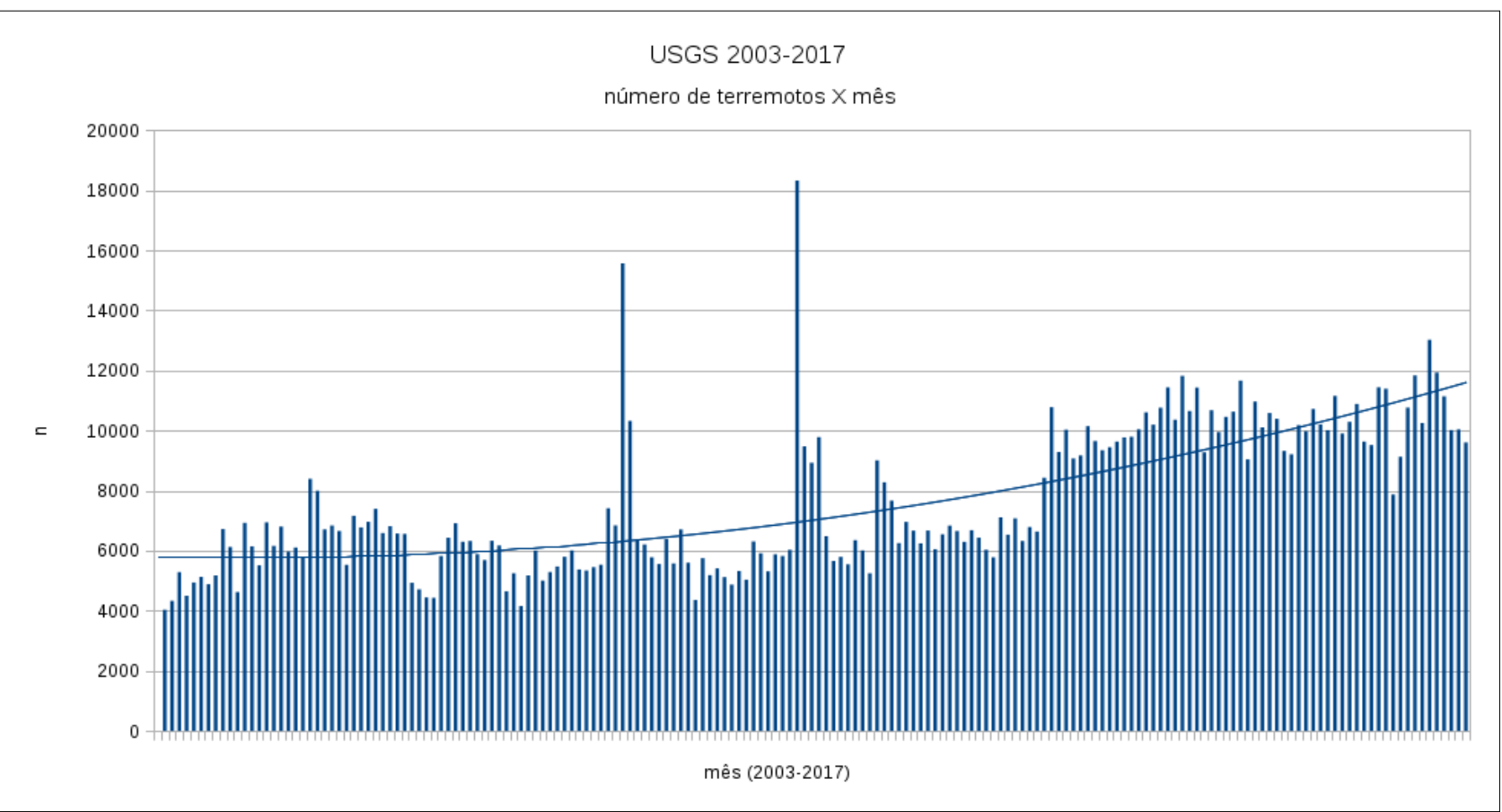

Fig. 5 - Distribuição mensal de terremotos na base USGS para o período 2003-2017; ainda que os dados sejam os mesmos, há, naturalmente, variação da escala do eixo de ordenadas e consequente alteração dos parâmetros que descrevem a curva quadrática representativa do conjunto, descrita abaixo.

$f(x)=0,21 x^{2}-5,62 x+5820,71$ 

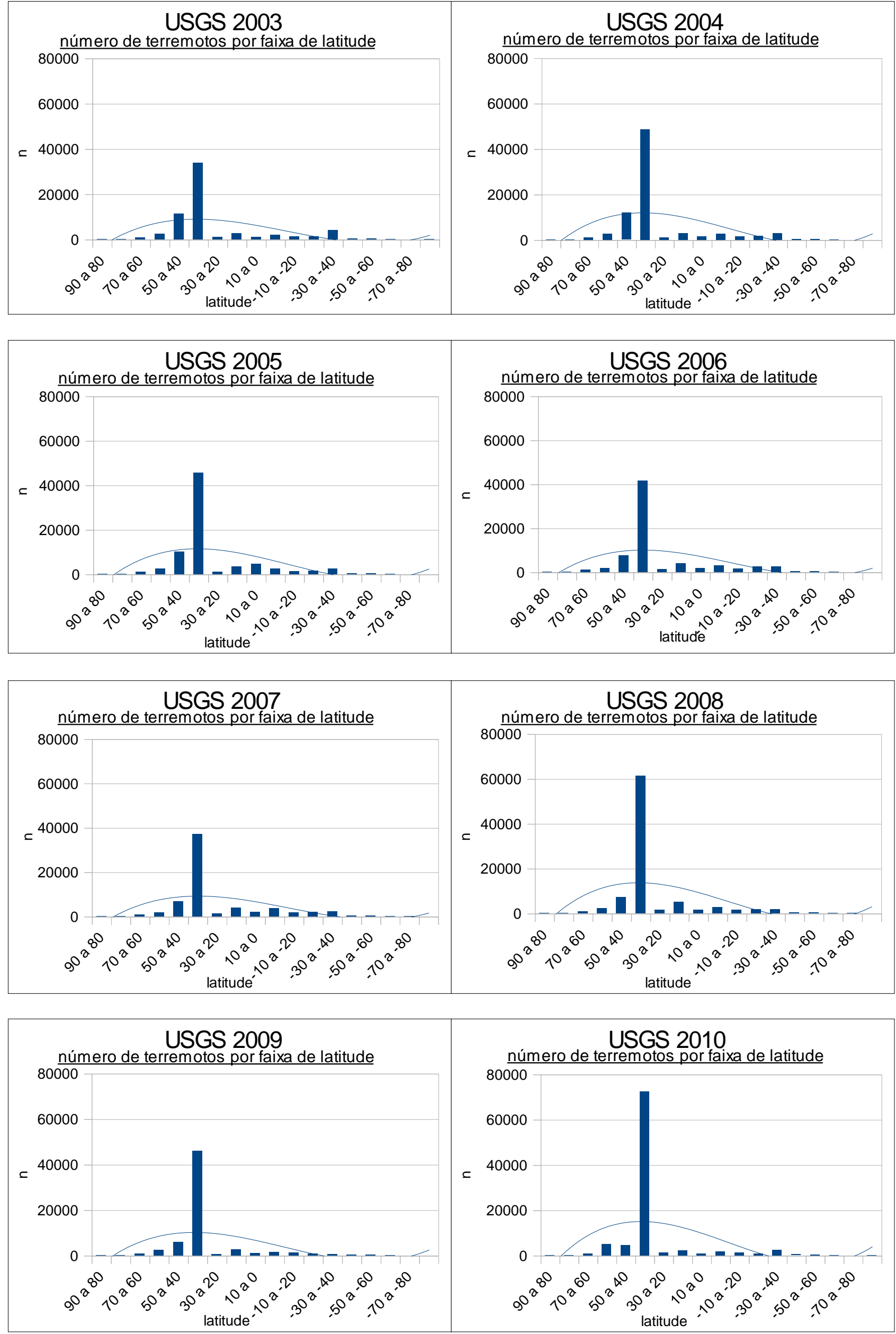

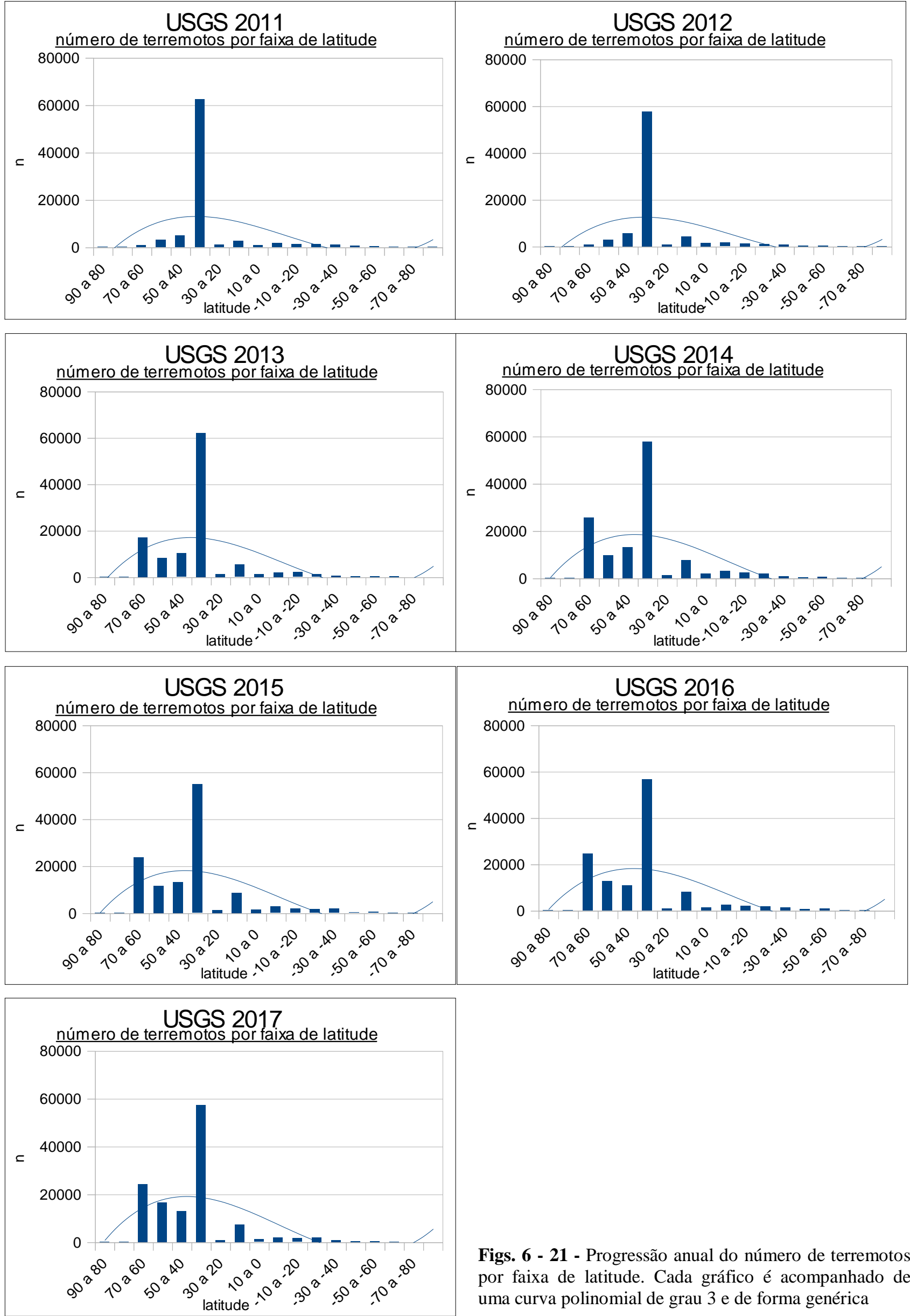

Figs. 6 - 21 - Progressão anual do número de terremotos por faixa de latitude. Cada gráfico é acompanhado de uma curva polinomial de grau 3 e de forma genérica 
Por certo, o perfil desses gráficos, absolutamente dominado pelos valores de $n$ na faixa de maior expressividade latitudinal, tem configuração muito semelhante em todo o conjunto, a qual é expressa pela relação geral

$f(x)=a x^{3}-b x^{2}+c x-d$

Ainda que a faixa $40^{\circ} \mathrm{N}-30^{\circ} \mathrm{N}$ seja a mais destacada nos gráficos, o período mostra flutuação significativa de $n$ no hemisfério N: não apenas essa faixa varia entre $\sim 33 \mathrm{~K}$ eventos $(2003)$ e $\sim 72 \mathrm{~K}$ eventos (máximo em 2010), mantendo-se sempre acima dos 54K eventos desde esse ano, mas também a larga faixa $70^{\circ} \mathrm{N}-40^{\circ} \mathrm{N}$ ganha mais atividade a partir de 2012 . As relações espaciais entre os prolongamentos tectônicos das zonas sísmicas compreendidas por essas faixas devem ser determinadas, mas com refinamento que extrapola o critério aqui adotado e segundo proposta que já vem sendo desenvolvida no âmbito do projeto em curso.

$\begin{array}{llllllllllllllll} & 2003 & 2004 & 2005 & 2006 & 2007 & 2008 & 2009 & 2010 & 2011 & 2012 & 2013 & 2014 & 2015 & 2016 & 2017 \\ 2003 & & 0,99 & 0,99 & 0,99 & 0,98 & 0,97 & 0,98 & 0,96 & 0,97 & 0,97 & \mathbf{0 , 9 4} & 0,90 & 0,90 & 0,89 & 0,89 \\ 2004 & 0,99 & & 1,00 & 1,00 & 1,00 & 0,99 & 0,99 & 0,98 & 0,99 & 0,99 & \mathbf{0 , 9 6} & 0,91 & 0,91 & 0,90 & 0,90 \\ 2005 & 0,99 & 1,00 & & 1,00 & 1,00 & 0,99 & 0,99 & 0,98 & 0,99 & 0,99 & \mathbf{0 , 9 6} & 0,91 & 0,91 & 0,90 & 0,90 \\ 2006 & 0,99 & 1,00 & 1,00 & & 1,00 & 1,00 & 1,00 & 0,99 & 0,99 & 0,99 & \mathbf{0 , 9 6} & 0,91 & 0,91 & 0,90 & 0,90 \\ 2007 & 0,98 & 1,00 & 1,00 & 1,00 & & 1,00 & 1,00 & 0,99 & 0,99 & 0,99 & \mathbf{0 , 9 6} & 0,90 & 0,90 & 0,90 & 0,89 \\ 2008 & 0,97 & 0,99 & 0,99 & 1,00 & 1,00 & & 1,00 & 1,00 & 1,00 & 1,00 & \mathbf{0 , 9 6} & 0,91 & 0,90 & 0,90 & 0,90 \\ 2009 & 0,98 & 0,99 & 0,99 & 1,00 & 1,00 & 1,00 & & 1,00 & 1,00 & 1,00 & \mathbf{0 , 9 6} & 0,91 & 0,91 & 0,91 & 0,90 \\ 2010 & 0,96 & 0,98 & 0,98 & 0,99 & 0,99 & 1,00 & 1,00 & & 1,00 & 1,00 & \mathbf{0 , 9 6} & 0,90 & 0,90 & 0,90 & 0,90 \\ 2011 & 0,97 & 0,99 & 0,99 & 0,99 & 0,99 & 1,00 & 1,00 & 1,00 & & 1,00 & \mathbf{0 , 9 6} & 0,90 & 0,90 & 0,90 & 0,90 \\ 2012 & 0,97 & 0,99 & 0,99 & 0,99 & 0,99 & 1,00 & 1,00 & 1,00 & 1,00 & & \mathbf{0 , 9 6} & 0,91 & 0,91 & 0,91 & 0,90 \\ 2013 & \mathbf{0 , 9 4} & \mathbf{0 , 9 6} & \mathbf{0 , 9 6} & \mathbf{0 , 9 6} & \mathbf{0 , 9 6} & \mathbf{0 , 9 6} & \mathbf{0 , 9 6} & \mathbf{0 , 9 6} & \mathbf{0 , 9 6} & \mathbf{0 , 9 6} & & 0,99 & 0,98 & 0,99 & 0,98 \\ 2014 & 0,90 & 0,91 & 0,91 & 0,91 & 0,90 & 0,91 & 0,91 & 0,90 & 0,90 & 0,91 & 0,99 & & 1,00 & 1,00 & 0,99 \\ 2015 & 0,90 & 0,91 & 0,91 & 0,91 & 0,90 & 0,90 & 0,91 & 0,90 & 0,90 & 0,91 & 0,98 & 1,00 & & 1,00 & 1,00 \\ 2016 & 0,89 & 0,90 & 0,90 & 0,90 & 0,90 & 0,90 & 0,91 & 0,90 & 0,90 & 0,91 & 0,99 & 1,00 & 1,00 & & 1,00 \\ 2017 & 0,89 & 0,90 & 0,90 & 0,90 & 0,89 & 0,90 & 0,90 & 0,90 & 0,90 & 0,90 & 0,98 & 0,99 & 1,00 & 1,00 & \end{array}$

Figura 22 - Correlações entre os dados médios anuais para o período 2002-2017 na base USGS. O ano de 2013, considerado transicional em função de seu comportamento estranho às duas frações mais homogêneas, foi destacado em cinza. 


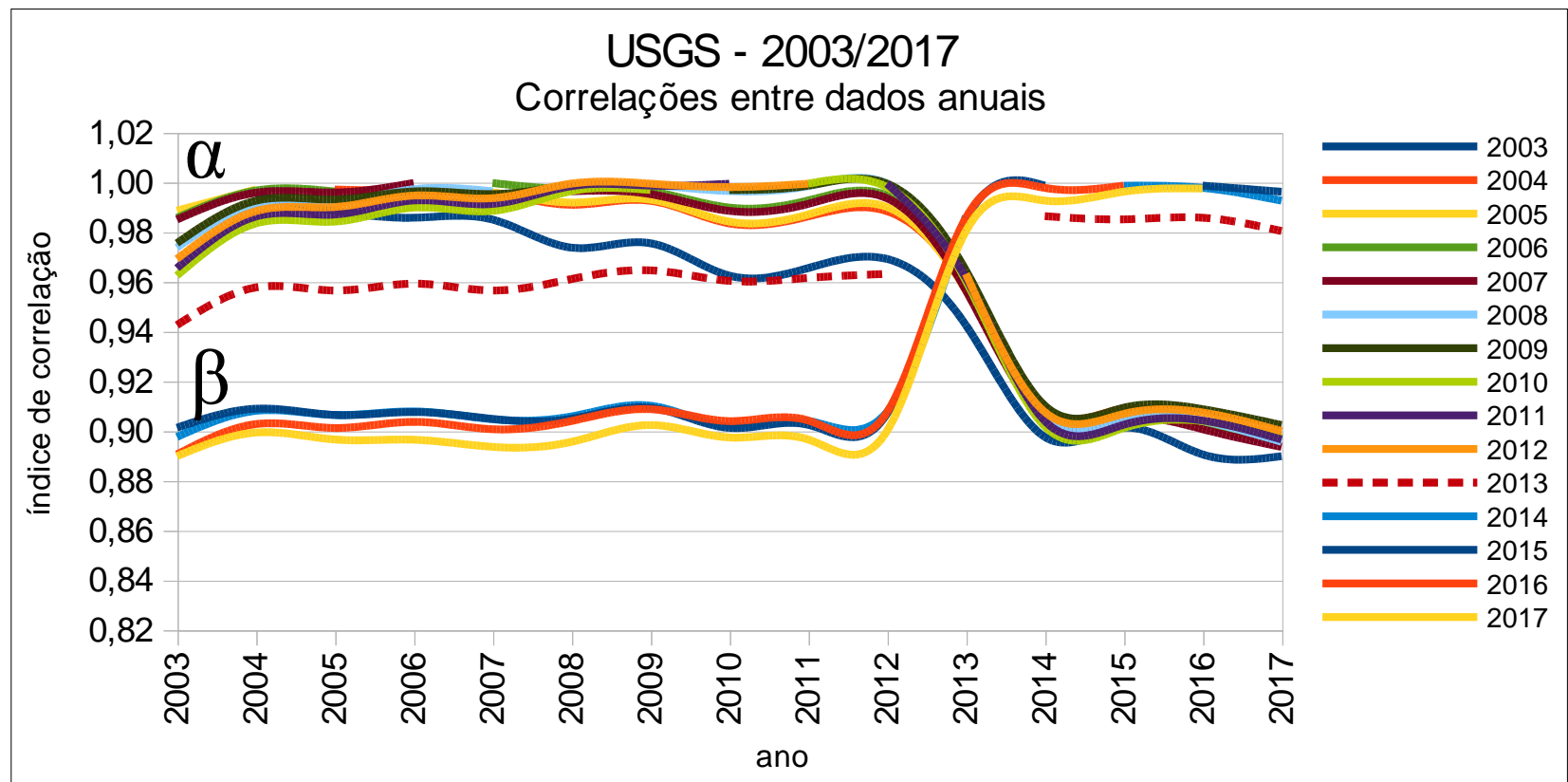

Figura. 23 - Perfis de correlação entre os dados brutos anuais apresentados nas figuras 7-22 acima, exibindo dois conjuntos separados pelo ano transicional de 2013 (tracejado). Ao refletirem as informações dispostas na Figura 23, as curvas são, obviamente, interrompidas.

\section{CONSIDERAÇÕES FINAIS}

Como já mencionado, este trabalho se restringe apenas à distribuição de terremotos segundo faixas de latitude; os resultados, portanto, fazem simplesmente referência à variação de frequência de sismos registrados na base USGS em faixas tectônicas de variadas características, e a eleição de critérios distintos, como magnitude e tipo de magnitude, certamente levará a outras considerações de cunho sismológico. Entretanto, mesmo considerando a) uma possível distorção numérica diante da presença de redes sismológicas de grandes densidade e sensibilidade na Europa, nos EUA e no Japão, que permitem a identificação de microssismos de diversas naturezas, e b) considerando-se o curto intervalo de amostragem, há inegável elevação da sismicidade em largas faixas do globo, como atestam os gráficos anuais. Em vista da disponibilidade de informações sobre mecanismos focais de terremotos - por exemplo, na base USGS -, um caminho promissor é, então, oferecido pela análise da sismicidade associada, em ambientes bem definidos, a diferentes regimes tensionais. Áreas de falhamento transformante ligadas a zonas de subducção ou a margens divergentes são um instigante exemplo ainda a ser estudado.

A correlação entre os conjuntos totais de dados anuais representados nos gráficos das Figuras 6 a 21 corrobora, uma vez mais, a existência de comportamentos distintos entre o início e o final do período de amostragem: a tabela e o gráfico reproduzidos nas Figuras 22 e 23 apresentam as correlações dos dados brutos anuais, o que permite verificar a eventual manutenção dos mesmos padrões de distribuição numérica de sismos em diferentes faixas de latitude. Percebe-se claramente a melhor correlação entre as distribuições anteriores a 2012, de um lado (assinalados, no gráfico, 
como $\alpha$ ), e as posteriores a 2014, de outro (assinalados, no gráfico, como $\beta$ ), o que indica, como já apontado, a instalação de um regime sísmico diferente a partir do ano transicional de 2013.

Observe-se, ainda, a coerência entre os gráficos correspondentes às Figuras 4, 5 e 23, que sugerem uma elevação do número de registros referentes à sismicidade mundial, independente da magnitude. Por certo, a explicação para esse aumento de frequência de terremotos não reside somente na sismologia, mas deve ser integrada no modelo geotectônico e inserida em bases de dados de maior amplitude cronológica (Bullen \& Bolt 1985:378); além disso, há que se desenvolver toda uma série de considerações sobre o mecanismo envolvido nos fenômenos sísmicos e em sua monitoração a fim de explicar de maneira adequada o padrão exibido no gráfico da Figura 23.

A análise de dados sísmicos a partir de métodos estatísticos permite não apenas atestar a consistência das informações disponíveis, mas também verificar a robustez da proposta de acompanhamento da sismicidade a partir de bases de dados. É imprescindível, diante disso, eleger um recorte espacial adequado, como o já sugerido ambiente tectônico, a fim de se obterem resultados mais coerentes com o modelo de dinâmica global.

\section{REFERÊNCIAS}

ABE, S.; SUZUKI, N. Law for the distance between successive earthquakes. Journal of Geophysical Research, v.108, n.B2, 2003.

BUlLEN, K. E.; BOLT, B. A. An introduction to the theory of seismology. Cambridge: University Press, 1985.

CHIARABBA, C.; JOVANE, L.; DiSTEFANO, R. A new view of Italian seismicity using 20 years of instrumental recording. Tectonophysics, v.395, n.3-4, p.251-268, 2005.

BALLORE, F. M. de. Les tremblements de terre: géographie séismologique. Paris: Libr. Armand Colin, 1906.

EKSTRÖM, G. Global seismicity: results from systematic waveform analyses, 1976-2005. In: KANAMORI, H. Earthquake seismology. NY: Elsevier, 2009.

GUGLIELMI, A.; ZOTOV, O. Impact of the Earth's oscillations on the earthquakes. arXiv:1207.0365v1 [physics.geoph], 2012.

GUTENBERG, B.; RICHTER, C.F. Seismicity of the Earth and associated phenomena. Princeton: University Press, 1949.

KAGAN, Yan Y. Earthquakes - models, statistics, testable forecasts. Oxford: John Wiley \& Sons, 2014.

KAGAN, Yan Y. Why does theoretical physics fail to explain and predict earthquake occurrence? In: BHATTACHARYYA, P.; CHAKRABARTI, B. K. (Eds). Modelling critical and catastrophic phenomena in Geoscience: A statistical physics approach. Berlin: Springer, 2006. 
McCAFFREY, R. Global frequency of magnitude 9 earthquakes. Geology, v. 36, p.263-266, 2008.

OGATA, Y;; KATSURA, K. Analysis of temporal and spatial heterogeneity of magnitude frequency distribution inferred from earthquake catalogues. Geophys. Journal Int., v.113, p. 727-738, 1993.

ROTHÉ, J. P. The seismicity of the Earth / La séismicité du globe 1953-1965. Paris: UNESCO, 1969.

SIDORENKOV, N. S. The interaction between Earth's rotation and geophysical processes. NY: John Wiley \& Sons, 2009.

TURCOTTE, D.L.; MALAMUD, B.D. Earthquakes as a complex system. In: LEE, W.H.K.; JENNINGS, P.; HISSLINGER, C.; KANAMORI, H. International handbook of earthquake and engineering seismology. NY: Elsevier, 2002. 